Алгебра и анализ

Том 22 (2009), № 1
St. Petersburg Math. J.

Vol. 22 (2011), No. 1, Pages 73-79

S 1061-0022(2010)01134-8

Article electronically published on November 17, 2010

\title{
A CLASS OF TOPOGRAPHICAL WAVEGUIDES
}

\author{
V. M. BABICH
}

\begin{abstract}
In the case of some infinite domains, it is shown that the spectrum of the elasticity theory operator is not purely continuous. This implies the existence of a new class of the so-called topographical waveguides.
\end{abstract}

\section{§1. INTRODUCTION}

Volcanic eruptions generate seismic waves of various types. In particular, waves have been observed that spread along "chainlets" of mountains formed by quite firm rocks in a softer, low-speed medium. Thus, a waveguide propagation of seismic waves has been detected 11 A mathematical description of this type of phenomena is a complicated unsolved problem. However, waveguide propagation of elastic waves has been studied in many papers - either of a computational nature, or done within a "physical" level of rigor, or pure mathematical. In the first place, the paper 1 should be mentioned, in which, "at the theorems level", the so-called topographical waveguides were treated. Such a waveguide is a narrow triangular or rectangular prism "attached" to an elastic half-space (see Figure 1).

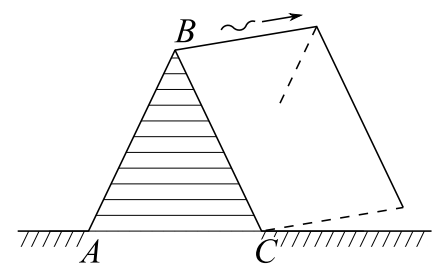

Figure 1

Next, there are papers devoted to the proof of the existence of a wave that runs along the edge of an elastic wedge; they are also in a close relationship with topographical waveguides. The existence of such a wave (whose waveguide essence is clear) was beyond any doubt already after the computational papers [2, 3, and the papers 4, 5, done on a "physical" level of mathematical rigor. However, a rigorous result is due to I. Kamotskiı (see [6), who employed a variational approach to prove the existence of a vector-valued eigenfunction corresponding to this wave mode, for the elasticity theory operator. In [6], a significant role was played by a clever choice of a test function.

Our aim in this paper is to consider new classes of topographical waveguides. We show that in a short-wave situation, an arbitrary elastic prism attached to an elastic half-space is a topographical waveguide provided that the upper dihedral angle $(\angle A B C$ in Figure 1) is acute. This result admits a series of generalizations (see $\S 5$ ). The problem

2010 Mathematics Subject Classification. Primary 35Q86, 74L05.

Key words and phrases. Waveguide, point spectrum, selfadjoint operator, elasticity theory equations. Supported by RFBR (grant no. 07-01-548).

${ }^{1}$ The author is indebted to Professor P. V. Krauklis for this information. 
reduces to the proof of the existence of a point spectrum for a certain vectorial selfadjoint differential operator.

In the constructions, a key role is played by some analytic tricks borrowed from [6].

\section{§2. Statement of the problem}

We assume that $\Xi$ is a domain in $\mathbb{R}^{3}=-\infty<x, y, z<+\infty$, described by the inequality $y<f(x)$, where $f(x)$ is a nonnegative continuous function defined for $-\infty<$ $x<+\infty$ and vanishing outside a finite interval $[a, b]$. Moreover, the graph of $f(x)$ for $a \leq$ $x \leq b$ is assumed to consist of two rectilinear segments $[a M]$ and $[M b](f(a)=f(b)=0$, $\left.\left.M=\left(x_{0}, f\left(x_{0}\right)\right), a<x_{0}<b, f\left(x_{0}\right)>0\right)\right)$ forming an acute angle at $M$. The domain $\Xi$ is a topological product: $\Xi=\Omega \times(-\infty<z<+\infty)$, where $\Omega$ is the domain $y<f(x)$ on the $(x, y)$-plane; see Figure 2 .

Let $U(t, x, y, z)=\left(U_{x}, U_{y}, U_{z}\right)$ be a solution of the elasticity theory equations in $\Xi$,

$$
\begin{aligned}
\rho U_{t t} & =(\lambda+\mu) \operatorname{grad} \operatorname{div} U+\mu \Delta U=0, \\
\rho, \lambda, \mu & =\text { const, } \quad \rho>0, \quad \mu>0, \quad \lambda+\frac{2}{3} \mu>0,
\end{aligned}
$$

that has the form

$$
U=e^{-i \omega t+i k z} u(x, y), \quad \omega, k=\text { const }, \omega>0, k>0,
$$

and possesses no strain on the boundary $\partial \Xi$. We arrive at the following equation for $u$ in $\Omega$ :

$$
\rho \omega^{2} u=L(i k) u
$$

where

$$
L(i k) u=-(\lambda+\mu) \operatorname{grad} \operatorname{div} U-\mu \Delta U .
$$

On the right in the last identity, the differentiation $\frac{\partial}{\partial z}$ is assumed to be replaced by multiplication by $i k$.

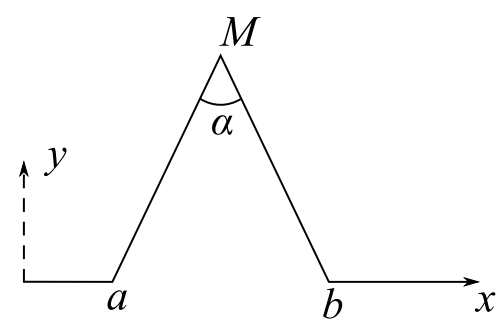

FiguRE 2

We subject the vector (2.2) to the boundary condition of the absence of strain on $\partial \Omega$ (i. e., for $y=f(x)$ ). We arrive at the identity

$$
N(i k) u=0,
$$

where $N(i k)$ is a first order differential operator depending on $k$.

Later, we shall describe the meaning of the equation and boundary conditions more precisely. We use the notation employed in [6]. In what follows, we put $\rho=1$, which leads to no loss of generality.

If a function $u \not \equiv 0$ satisfies equation (2.3) and the boundary conditions (2.4), and is square integrable over $\Omega$ (i. e., $u$ is a vector-valued eigenfunction of the operator $L(i k)$ that corresponds to the boundary conditions (2.4)), then the vector-valued function (2.2) is called a wave mode. The proof of the existence of wave modes is the subject of the present paper. 
We state the problem in more precise terms 2 On smooth (in $C^{\infty}(\bar{\Omega})$ ) vector-valued functions $u$ (with values in $\mathbb{C}^{3}$ ) having bounded support and satisfying the boundary conditions (2.4), the operator $L(i k)$ is positive and symmetric in the Hilbert space of square integrable vector-valued functions. In what follows, by $L(i k)$ we mean the extension of the above operator up to a Friedrichs selfadjoint operator. We shall prove the existence of a point spectrum for this extension. Namely, the following will be established.

Theorem 1. For $k$ sufficiently large, $L(i k)$ has at least one eigenvalue on the interval $\left(0, c_{R}^{2} k^{2}\right)$.

\section{§3. VARIATIONAL APPROACH TO THE PROOF}

The strategy of the existence proof for a point spectrum is fairly standard; see [1, 6]. It is well known (see [1]) that the essential spectrum of the operator $L(i k)$ occupies the half-axis $\left[c_{R}^{2} k^{2},+\infty\right)$, where $c_{R}$ is the Rayleigh wave velocity.

The lower bound of the spectrum is

$$
\inf \Phi(u), \Phi(u):=\frac{a_{\Omega}(i k, u, u)}{(u, u)}, \quad u \in H^{1}(\Omega), u \neq \equiv,
$$

where $a_{\Omega}$ is the quadratic form of the operator $L(i k)$ and $(u, u)$ is the square of the usual $L_{2}$-norm of $u$. In order to prove the existence of a point spectrum, it suffices to find a function $u^{\text {test }}$ with

$$
\Phi\left(u^{\text {test }}\right)<c_{R}^{2} k^{2} .
$$

To construct this test function, we place the coordinate system as shown in Figure 3 . On the interval $[0, d]$, let the part of $\partial \Omega$ not lying on the $x_{1}$-axis be the rectilinear segment described by the equation $x_{2}=x_{1} \tan \alpha, 0<\alpha<\frac{\pi}{2}$ (Figure 3).

We denote by $U^{R}$ the expression (2.2) that describes the classical Rayleigh wave propagating in the direction of the $x_{3}$ axis. Strain is assumed to be absent for $x_{2}=0$. Then

$$
U^{R}=e^{-i \omega t+i k x_{3}} u^{R}\left(x_{2}\right),
$$

where $u^{R}$ does not depend on $x_{1}$ and $x_{3}$. Consider the following cutoff function $\eta\left(x_{1}\right)$ defined for $x_{1} \geq 0$ :

$$
\begin{aligned}
& \eta\left(x_{1}\right)= \begin{cases}1 & \text { if } 0 \leq x_{1}<d^{\prime} \\
0 & \text { if } x_{1}>d\end{cases} \\
& 0<d^{\prime}<d, \quad d, d^{\prime}=\text { const }, \quad \eta \geq 0, \quad \frac{d \eta}{d x} \leq 0, \quad \eta \in C^{\infty}\left(\mathbb{R}^{+}\right) .
\end{aligned}
$$

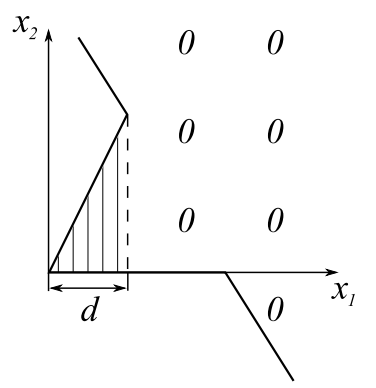

FigURE 3

\footnotetext{
${ }^{2}$ The problems treated in [1] and [6] were introduced similarly.
} 
The test function can be chosen as follows (cf. [6, formula (3.12)]):

$$
u_{k}^{\text {test }}\left(x_{1}, x_{2}\right)=: \begin{cases}\eta\left(x_{1}\right) u^{R}\left(x_{2}\right) & \text { in } \Omega_{1}, \\ 0 & \text { in } \Omega \backslash \Omega_{1} .\end{cases}
$$

Here $\Omega_{1}$ is the triangle

$$
0 \leq x_{1} \leq d, \quad 0 \leq x_{2} \leq x_{1} \tan \alpha
$$

(see Figure 3).

Easy considerations to be described in the next section show that

$$
\Phi\left(u_{k}^{\text {test }}\right)=c_{R}^{2} k^{2}-\text { const } k+O(1), \quad \text { const }>0,
$$

as $k \rightarrow+\infty$. But (3.7) implies (3.2) for $k$ sufficiently large. This proves the theorem (see the end of $\S 2$ ).

\section{$\S 4$. THE PROOF OF $(3.7)$}

First, we observe that

$$
a_{\Omega}(i k, u, u)=\int_{\Omega} a(i k, u, u) d x_{1} d x_{2},
$$

where $a$ is an expression quadratic in $u$ and its first derivatives, specifically,

$$
a=\lambda \operatorname{div} u(\operatorname{div} u)^{*}+\frac{\mu}{2} \frac{\partial u_{l}}{\partial x^{m}}\left(\frac{\partial u_{m}}{\partial x^{l}}\right)^{*}+\frac{\mu}{2} \frac{\partial u_{l}}{\partial x^{m}}\left(\frac{\partial u_{l}}{\partial x^{m}}\right)^{*}
$$

$\left(\frac{\partial}{\partial x_{3}}\right.$ must be replaced by multiplication by $i k$, where the star indicates complex conjugation).

Clearly,

$$
\Phi\left(u_{k}^{\mathrm{test}}\right)=\frac{\int_{\Omega_{1}} a\left(i k, u_{k}^{\mathrm{test}}, u_{k}^{\mathrm{test}}\right) d x_{1} d x_{2}}{\int_{\Omega_{1}} u_{k}^{\text {test }} u_{k}^{* \text { test }} d x_{1} d x_{2}}, \quad u_{k}^{\text {test }} u_{k}^{* \text { test }}=\sum_{j=2}^{3} u_{k, j}^{\text {test }} u_{k, j}^{* \text { test }} .
$$

This can be transformed to

$$
\begin{aligned}
\Phi\left(u_{k}^{\mathrm{test}}\right)=c_{R}^{2} k^{2} & -\frac{\int_{0}^{d} \eta^{2}\left(x_{1}\right)\left(c_{R}^{2} k^{2} \int_{0}^{x_{1} \tan \alpha} u^{R} u^{* R} d x_{2}-\int_{0}^{x_{1} \tan \alpha} a\left(i k, u^{R}, u^{* R}\right) d x_{2}\right) d x_{1}}{\int_{0}^{d} \eta^{2}\left(x_{1}\right) \int_{0}^{x_{1} \tan \alpha} u^{R} u^{* R} d x_{2} d x_{1}} \\
& +\mu \frac{\int_{0}^{d}\left(\frac{d \eta}{d x_{1}}\right)^{2} d x_{1}}{\int_{0}^{d} \eta^{2}\left(x_{1}\right) d x_{1}}=A_{1}-A_{2}+A_{3},
\end{aligned}
$$

where $\mu$ is the shift module.

The summand $A_{3}$ does not depend on $k$, so $A_{3}=O(1)$ as $k \rightarrow+\infty$. The expression $A_{2}$ is positive because always

$$
c_{R}^{2} k^{2} \int_{0}^{N} u^{R} u^{* R} d x_{2}>\int_{0}^{N} a\left(i k, u^{R}, u^{R}\right) d x_{2}, \quad N>0
$$

(see [6]). We find the asymptotics of $A_{2}$ as $k \rightarrow+\infty$. First, recall that, under a due choice of a scalar factor, we have

$$
u^{R}=\alpha_{1} e^{-a_{1} k x_{2}}+\alpha_{2} e^{-a_{2} k x_{2}}, \quad a_{1}, a_{2}=\text { const }>0 .
$$


Here $\alpha_{1}=\left(0, \alpha_{11}, \alpha_{12}\right)$ and $\alpha_{2}=\left(0, \alpha_{21}, \alpha_{22}\right)$ are constant nonzero vectors. From (4.6) it follows that

$$
\begin{gathered}
\psi_{0}\left(k x_{2}\right):=u^{R} u^{* R}=\sum_{l, m=2}^{3} f_{l m} e^{-\beta_{l m} k x_{2}} \\
k^{2} \psi_{1}\left(k x_{2}\right):=k^{2} c_{R}^{2} u^{R} u^{* R}-a\left(i k, u^{R}, u^{* R}\right)=k^{2} \sum_{l, m=2}^{3} g_{l m} e^{-\beta_{l m} k x_{2}},
\end{gathered}
$$

where the $\beta_{l m}$ are positive constants and the $f_{l m}, g_{l m}$ are complex constants.

The expression $A_{2}$ is a fraction whose numerator is

$$
\int_{0}^{d} \eta^{2}\left(x_{1}\right) \int_{0}^{x_{1} \tan \alpha} k^{2} \psi_{1}\left(k x_{2}\right) d x_{2} d x_{1} .
$$

We find the asymptotics of (4.9) as $k \rightarrow+\infty$. Observe that

$$
\int_{0}^{x_{1} \tan \alpha} k^{2} \psi_{1}\left(k x_{2}\right) d x_{2}=k \int_{0}^{k x_{1} \tan \alpha} \psi_{1}(\xi) d \xi=k \psi_{2}\left(k x_{1}\right)
$$

By (4.5), here $\psi_{2}(\xi)$ is a positive function. Since

$$
\int_{0}^{\infty} k^{2} \psi_{1}\left(k x_{2}\right) d x_{2}=0
$$

we have

$$
\psi_{2}\left(k x_{1}\right)=\int_{0}^{+\infty} \psi_{1}(\xi) d \xi-\int_{k x_{1} \tan \alpha}^{\infty} \psi_{1}(\xi) d \xi=-\int_{k x_{1} \tan \alpha}^{\infty} \psi_{1}(\xi) d \xi .
$$

Together with (4.8), this implies that $\psi_{2}(\xi)$ is exponentially small as $\xi \rightarrow+\infty: \psi_{2}(\xi)=$ $O\left(e^{-\varepsilon \xi}\right), \varepsilon=$ const $>0$. Now it is easy to find the asymptotics of (4.9) as $k \rightarrow+\infty$. Indeed,

$$
\int_{0}^{d} \eta^{2}\left(x_{1}\right)\left(\int_{0}^{x_{1} \tan \alpha} k^{2} \psi_{1}\left(k x_{2}\right) d x_{2}\right) d x_{1}=\int_{0}^{d} \eta^{2}\left(x_{1}\right) k \psi_{2}\left(x_{1} k\right) d x_{1}=\int_{0}^{d^{\prime}}+\int_{d^{\prime}}^{d} .
$$

Since $\psi_{2}(\xi)$ is exponentially small as $\xi \rightarrow+\infty$, the second summand is also exponentially small, and we have

$$
\begin{array}{r}
\int_{0}^{d^{\prime}} k \psi_{2}\left(k x_{1}\right)=\int_{0}^{k d^{\prime}} \psi_{2}(\xi) d \xi=\int_{0}^{+\infty}-\int_{k d^{\prime}}^{+\infty}=\int_{0}^{+\infty} \psi_{2}(\xi) d \xi+O\left(e^{-k \varepsilon_{1}}\right) \\
\varepsilon_{1}=\text { const }>0 .
\end{array}
$$

Now, we analyze the denominator of the fraction $A_{2}$. By (4.7), it can be represented in the form

$$
\int_{0}^{d} \eta^{2}\left(x_{1}\right)\left(\int_{0}^{x_{1} \tan \alpha} u^{R} u^{* R} d x_{2}\right) d x_{1}=\int_{0}^{d} \eta^{2}\left(x_{1}\right)\left(\int_{0}^{x_{1} \tan \alpha} \psi_{0}\left(k x_{2}\right) d x_{2}\right) d x_{1} .
$$

For the inner integral, we have

$$
\int_{0}^{x_{1} \tan \alpha} \psi_{0}\left(k x_{2}\right) d x_{2}=\frac{1}{k} \int_{0}^{k x_{1} \tan \alpha} \psi_{0}(\xi) d \xi:=\frac{1}{k} \psi_{3}\left(k x_{1}\right),
$$

where $\psi_{3}(\xi)$ is a monotone increasing function. By (4.7), the $\operatorname{limit}_{\lim _{\xi \rightarrow+\infty}} \psi_{3}(\xi):=$ $\psi_{3}(+\infty)$ is finite: $0<\psi_{3}(+\infty)<+\infty$. We return to (4.13). Taking (4.4) into account, 
we can write

$(4.15)$

$\int_{0}^{d} \eta^{2}\left(x_{1}\right)\left(\int_{0}^{x_{1} \tan \alpha} \psi_{0}\left(k x_{2}\right) d x_{2}\right) d x_{1}=\int_{0}^{d^{\prime}} \frac{1}{k} \psi_{3}\left(k x_{1}\right) d x_{1}+\int_{d^{\prime}}^{d} \eta^{2}\left(x_{1}\right) \frac{1}{k} \psi_{3}\left(k x_{1}\right) d x_{1}$.

Next, $\psi_{3}(\xi)=\psi_{3}(+\infty)+O\left(e^{-\xi \varepsilon_{2}}\right)$ as $\xi \rightarrow+\infty, \varepsilon_{2}=$ const $>0$.

From (4.15), we deduce the following asymptotic relation:

$$
\int_{0}^{d} \eta^{2}\left(x_{1}\right)\left(\int_{0}^{x_{1} \tan \alpha} \psi_{0}\left(k x_{2}\right) d x_{2}\right) d x_{1}=\frac{1}{k} \psi_{3}(+\infty) \int_{0}^{d} \eta^{2}\left(x_{1}\right) d x_{1}+O\left(\frac{1}{k^{2}}\right) .
$$

Taken together, (4.12) and (4.16) easily give an asymptotic formula for $A_{2}$ (see (4.4)):

$$
A_{2}=k\left(\frac{\int_{0}^{+\infty} \psi_{2}(\xi) d \xi}{\psi_{3}(+\infty) \int_{0}^{d} \eta^{2}\left(x_{1}\right) d x_{1}}+O\left(\frac{1}{k^{2}}\right)\right)
$$

Since $A_{3}=O(1)$ as $k \rightarrow+\infty$, we arrive at the following asymptotic formula for $\Phi\left(u_{k}^{\text {test }}\right)$ $(\operatorname{see}(3.2))$ :

$$
\Phi\left(u_{k}^{\text {test }}\right)=A_{1}-A_{2}+A_{3}=k^{2} c_{R}^{2}-k \frac{\int_{0}^{+\infty} \psi_{2}(\xi) d \xi}{\psi_{3}(+\infty) \int_{0}^{d} \eta^{2}\left(x_{1}\right) d x_{1}}+O(1) .
$$

For large $k$, formula (4.18) implies (3.2), which proves Theorem 1 of $\S 2$.

\section{§5. ReFinements And ANAlogs of the RESUlt obtained}

Note that the function $u_{k}^{\text {test }}$ vanishes in $\Omega$ everywhere outside the dashed "triangle" in Figure 3. Therefore, certain analogs of Theorem 1 remain true for a wide class of domains in $\mathbb{R}^{2}$. It suffices merely to suppose that the boundary contains a "small angle" such as $\angle A B C$ in Figure 1 and the essential spectrum occupies the half-axis $\left[k^{2} c_{R}^{2},+\infty\right)$. Examples of such domains are shown in Figures 4, 5, and 6.

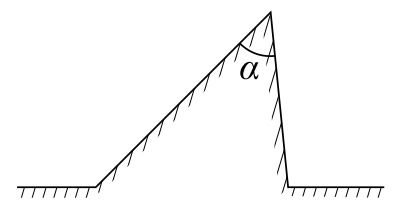

FigURE 4

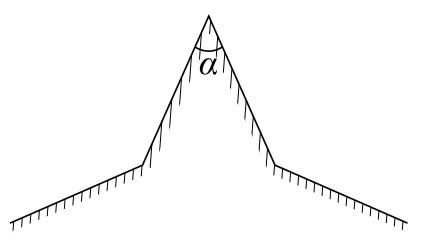

FIGURE 5

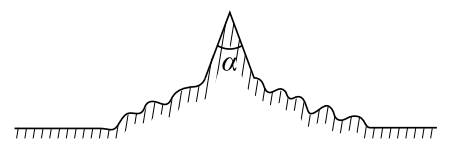

Figure 6 


\section{REFERENCES}

[1] A. S. Bonnet-Ben Dhia, J. Duterte, and P. Joly, Mathematical analysis of elastic surface waves in topographic waveguides, Math. Models Methods Appl. Sci. 9 (1999), no. 5, 755-798. MR1697393 (2000d:74036)

[2] A. A. Maradudin et al., Variational edge modes in finite crystals, Phys. Rev. B 6 (1972), 1106-1111.

[3] P. E. Lagasse, Analysis of dispersion-free guides for elastic waves, Electron. Lett. 8 (1972), no. 15, $372-373$.

[4] V. V. Krylov, Geometrical-acoustic approach to the description of localized modes of vibration of an elastic wedge, Zh. Tekhn. Fiz. 60 (1990), no. 2, 1-7. (Russian)

[5] A. V. Shanin, Excitation and scattering of a wedge wave in an obtuse elastic wedge close to $180^{\circ}$, Akust. Zh. 43 (1997), no. 3, 402-408. (Russian)

[6] I. V. Kamotski1̌, On a surface wave traveling along the edge of an elastic wedge, Algebra i Analiz 20 (2008), no. 1, 86-92; English transl., St. Petersburg Math. J. 20 (2009), no. 1, 59-63. MR2411969 (2009d:74042)

St. Petersburg Branch, Steklov Mathematical Institute, 27 Fontanka, St. Petersburg 191023, RUsSiA

E-mail address: babich@pdmi.ras.ru

Received 2/SEP/2009

Translated by S. V. KISLYAKOV 